\title{
Structural and immunological characterization of an epitope within the PAN motif of ectodomain I in Babesia bovis apical membrane antigen 1
}

\section{Amarin Rittipornlertrak}

Chiang Mai University Faculty of Veterinary Medicine

Boondarika Nambooppha

Chiang Mai University Faculty of Veterinary Medicine

Anucha Muenthaisong

Chiang Mai University Faculty of Veterinary Medicine

Veerasak Punyapornwithaya

Chiang Mai University Faculty of Veterinary Medicine

\section{Saruda Tiwananthagorn}

Chiang Mai University Faculty of Veterinary Medicine

\section{Yang-Tsung Chung}

National Chung Hsing University College of Veterinary Medicine

\section{Bumduuren Tuvshintulga}

Obihiro Chikusan Daigaku

Thillaiampalam Sivakumar

Obihiro Chikusan Daigaku

Naoaki Yokoyama

Obihiro Chikusan Daigaku

Nattawooti Sthitmatee ( $\nabla$ drneaw@gmail.com )

Chiang Mai University Faculty of Veterinary Medicine https://orcid.org/0000-0002-2329-8802

Research article

Keywords: apical membrane antigen 1, Babesia bovis, invasion-inhibition, growth-inhibition, synthetic peptide

Posted Date: October 8th, 2020

DOI: https://doi.org/10.21203/rs.3.rs-69365/v1

License: (c) (1) This work is licensed under a Creative Commons Attribution 4.0 International License.

Read Full License 


\section{Abstract \\ Background}

Bovine babesiosis caused by Babesia bovis (B. bovis) affects the cattle industry worldwide with high mobility and mortality. Live-attenuated vaccines are currently used in some of the endemic countries, but their wide use is limited due to various reasons. Although recombinant vaccines have been proposed as an alternative to the live vaccines, such vaccines are not commercially available to date. Apical membrane antigen-1 (AMA-1) is one of the leading candidates for vaccine development against diseases caused by apicomplexan parasite species. In this study, we predicted an epitope from the plasminogen, apple and nematode (PAN) motif of domain I in the B. bovis AMA-1 (BDAMA-1) using a combination of linear and conformational B-cell epitope prediction software. The selected epitope was bioinformatically analyzed, synthesized as a peptide (sBbAMA-1), and then used to immunize a rabbit.

\section{Results}

The anti-sBbAMA-1 serum obtained was evaluated for its growth- and invasion-inhibitory effects on $B$. bovis merozoites in vitro. Our results demonstrated that the predicted BbAMA-1 epitope, which is located on surface-exposed a-helix of PAN motif in domain I at the apex area, elicits antibodies capable of recognizing the native BbAMA-1 in immunoassays. Importantly, as compared to the control groups, the rabbit anti-sBbAMA-1 serum at dilution of 1:5 significantly inhibited $(p<0.05)$ the growth of $B$. bovis merozoites by approximately $50-70 \%$ on day 3 and 4 of cultivation and the invasion of merozoites by approximately $60 \%$ within $4 \mathrm{~h}$ of incubation.

\section{Conclusion}

Our results indicate the epitope predicted from the PAN motif of BbAMA-1 domain I is neutralizationsensitive and may serve as a target antigen for vaccine development against bovine babesiosis caused by B. bovis.

\section{Background}

Bovine babesiosis caused by Babesia bovis (B. bovis), which is transmitted by the cattle tick (Rhipicephalus microplus), affects the cattle industry worldwide with high mobility and mortality [1]. The asexual multiplication of $B$. bovis merozoites in bovine red blood cells (RBCs) results in a hemolytic anemia. In addition, the adherence of parasitized RBCs to the endothelial cells of capillaries of brain and lungs is manifested as respiratory and nervous signs, leading to a fatal form of babesiosis known as cerebral babesiosis [2]. In general, control of bovine babesiosis, including that caused by B. bovis, is based on tick control, chemotherapy, and vaccination [2-3]. With the reports on the development of 
acaricide resistance in ticks and drug resistance in B. bovis [4], vaccination is considered to be the efficient control method [5].

Currently, live attenuated vaccines are used to immunize the cattle against $B$. bovis in some of the endemic countries [2]. However, limitations, such as vaccine breakthroughs due to strain variations, contamination with other blood-borne pathogens, and time-consuming production systems, prevent the wide use of such vaccines [2,6-8]. Therefore, the use of novel vaccines, such as recombinant vaccines, are proposed to overcome these limitations [9]. In the last decade, several B. bovis antigens have been studied as candidates for the development of recombinant vaccines. Among them, the apical membrane antigen 1 (AMA-1), which is a microneme protein (MIC) that had been extensively studied as a promising malaria vaccine candidate [10], was identified as a neutralization-sensitive antigen in $B$. bovis [11]. The exact functional role of AMA-1 is still unknown. However, the previous investigations suggested that the AMA-1 plays an important role in the invasion of Plasmodium falciparum ( $P$. falciparum) merozoites into RBCs [12-13]. The AMA-1 forms moving junctions between the merozoite and the RBC, and thereby facilitating the invasion of merozoite into the RBCs [14]. Importantly, antibodies to $P$. falciparum AMA-1 (PfAMA-1) detected native antigens in immunoassays and inhibited the growth and invasion of merozoites in vitro [15-17]. In immunized monkeys, AMA-1-induced protection against $P$. falciparum blood stage infection was superior to the protection provided by several other antigens [18-19]. Remarkably, the result from human trials showed that the AMA-1 formulated in different adjuvants was safe, well-tolerated and immunogenic [20-22].

However, the use of AMA-1 in malaria vaccine formulations is constrained due to its high genetic diversity [23-24]. In contrast, the B. bovis AMA-1 (BbAMA-1) is highly conserved in B. bovis [25-26], and therefore is a target for diagnostic assays [27]. The BbAMA-1 composes of three domains of ectodomain (domain I-III) [11]. Previous studies demonstrated that the antibodies raised against a recombinant protein encoding the conserved central region and against peptides synthesized based on epitopes in domain II and III, and N-terminal region of domain I significantly inhibited the in vitro growth and invasion of $B$. bovis merozoites $[11,28]$. However, epitopes in the plasminogen, apple and nematode (PAN) motif of BbAMA-1 were not yet investigated for their vaccine potential. In $P$. falciparum, several antibodies against epitopes in PAN motif of PfAMA-1 domain I inhibited the parasite growth significantly [29-32]. Therefore, we hypothesized that the epitopes in PAN motif of BbAMA-1 domain I are neutralization-sensitive.

To test this hypothesis, in the present study, we predicted an epitope in the PAN motif of BbAMA-1 domain I, using the linear and conformational B-cell epitope prediction softwares [26]. The predicted epitope was synthesized, and the resultant synthetic peptide (sBbAMA-1) was used to immunize a rabbit. Subsequently, the effect of obtained rabbit antiserum on the growth and invasion of $B$. bovis merozoites was evaluated in vitro compared to control group.

\section{Results}

\section{A potential epitope of BbAMA-1 predicted in this study}


Based on the BbAMA-1 sequence from the Texas strain of B. bovis, an epitope, KTRGSSSVTAAKLSPVSAKDLRRWGYEGNDVANCSEYASNLIPASDKTTK, located between the residues 181 and 230, was predicted within the PAN motif of ectodomain I using a combination of immunoinformatics tools. Multiple alignment of AMA-1 sequences from different $B$. bovis isolates identified 6 polymorphic sites at residues 188, 189, 199, 221, 227 and 228 (Fig. 1A). Homology modelling indicated that BbAMA-1 is structurally most similar to Babesia divergens AMA-1 (BdAMA-1) (PDB: 4apm) with 65\% identity, 56\% coverage and 0.558 of TM-score. Analysis of the BbAMA-1 protein structure revealed that the epitope residues localized at the apex region of BbAMA-1 (Fig. 2A). According to the 3D model and predicted secondary structure of BbAMA-1, this epitope forms a surface-exposed a-helix of the PAN motif of BbAMA-1 domain I (Fig. 2B). In P. falciparum, the invasion-inhibitory peptide R1 can inhibit the interaction between AMA-1 (PfAMA1) and rhoptry neck proteins (RON) (PfRON2), and thereby inhibiting the merozoite invasion [31]. Interestingly, I-TASSER modelling indicated that the ligand-binding site of the invasion-inhibitory peptide R1 (PDB: 3sriB) is associated with the predicted epitope on BbAMA-1 (Fig. 2C). The possible binding sites for invasion-inhibitory peptide R1 in our epitope are residues 204, 219, 220 , 221, 222, 223, 224, 229 and 230, as shown in Fig. 1B.

\section{Recognition of native protein by anti-sBbAMA-1 antibody}

In Western blot, the rabbit antiserum against sBbAMA-1 reacted with native protein sized approximately 82-, 69-, and 45-kDa in the parasite lysate but not in normal RBCs lysates (Fig. 3A). Furthermore, the antisBbAMA-1 antibody reacted strongly with $B$. bovis merozoites in IFAT, while no reactivity was observed when negative control serum was used (Fig. 3B). These results showed that the anti-sBbAMA-1 antibody was able to recognize the native BbAMA-1, confirming the antigenicity of the predicted B-cell epitope.

\section{In vitro growth-inhibitory effect and morphology changes}

To investigate the growth inhibitory effect of anti-sBbAMA-1 antibody, B. bovis was cultivated in media containing rabbit anti-sBbAMA-1 serum at 1:5, 1:10, and 1:20 dilutions, and the parasitemia was monitored daily. The findings showed that the anti-sBbAMA-1 antibody inhibited the parasite growth in a dilution-dependent manner, i.e., the dilutions of 1:5 had higher inhibitory effect than the dilutions of 1:10 and 1:20, respectively (Fig. 4). The rates of growth inhibition induced by anti-sBbAMA-1 serum at 1:5 dilution were $>50 \%$ and $>70 \%$ on days 3 and 4 post-cultivation, respectively. In addition, the parasite growth was significantly impeded at 1:10 and 1:20 anti-sBbAMA-1 dilutions on day 4 of cultivation.

The microscopic examination of Giemsa-stained blood smears prepared from anti-sBbAMA-1 serumtreated cultures revealed a gradual increase in the number of extracellular merozoites and pyknotic forms, compared to control cultures (Fig. 5). In particular, these changes were prominent in the cultures that contained anti-sBbAMA-1 serum at 1:5 dilution.

\section{In vitro invasion-inhibitory effect}


To investigate the effect of anti-sBbAMA-1 antibody on the merozoite invasion into RBCs, purified free merozoites isolated from $B$. bovisin vitro cultures were incubated together with fresh bovine RBCs in culture medium containing anti-sBbAMA-1 serum at 1:5 and 1:10, and the parasitemia was monitored at 1,2 and $4 \mathrm{~h}$. The anti-sBbAMA-1 serum had a dilution-dependent effect on the invasion of merozoites into the RBCs. We found that, as compared to GIT-control, the merozoite invasion was significantly inhibited ( $p$ $<0.05$ ) by approximately $60 \%$ in the cultures that contained anti-sBbAMA- 1 serum at $1: 5$ dilution after $4 \mathrm{~h}$ of incubation (Fig. 6). Furthermore, the invasion-inhibition caused by $1: 10$ and 1:20 dilutions at $2 \mathrm{~h}$ and 4 h was approximately $45 \%$, which was significantly higher compared with the negative control culture $(p<$ 0.05).

\section{Discussion}

In P. falciparum, the PAN domain is presumably known to mediate adhesion of other proteins or carbohydrate receptors to AMA-1 [33]. The PAN motif of PfAMA-1 domain I has been previously identified as the major target of 1F9 monoclonal antibody (MAb 1F9) (residue 191 to 247) [29-30,34] and human anti-AMA1 antibody (humAbAMA1) (residue 194 to 206) [32] and as the partial target of the invasioninhibitory peptide R1 [31, 35-37]. Here, we predicted an epitope from the PAN motif in BbAMA-1 ectodomain I and evaluated its vaccine potential. Interestingly, the sequence alignment of PAN motif of PfAMA-1 domain I and the corresponding region from BbAMA-1 demonstrated that the BbAMA-1 epitope extends along the conformational epitope region of PfAMA-1 [38] and the ligand-binding site of the invasion-inhibitory peptide $\mathrm{R} 1$. These results imply a possibility that the selected BbAMA-1 epitope, which is a target of immune recognition, induce a protective immunity against $B$. bovis infection.

In the present study, the rabbit antiserum from a rabbit immunized with the synthetic form of the selected epitope reacted positively with $B$. bovis merozoites in IFAT suggesting that the epitope elicits antibodies capable of recognizing the native AMA-1. In Western blot, in agreement with Gaffar et al. (2004) [11], the rabbit anti-sBbAMA-1 antibody reacted specifically with an approximately $82-k D a$ of $B$. bovis protein, representing an unprocessed full-length AMA-1. Moreover, in consistent with the findings from a previous study [28], we detected two additional bands at 69- and 45-kDa, which were not reported by Gaffar et al. (2004) [11]. The use of the antibodies that targeted different regions of the BbAMA-1 might be the reason for this discrepancy [28]. The proteolytic processing of AMA-1 upon merozoite invasion, as demonstrated in several apicomplexan species [39-42], might explain the multiple bands.

The AMA-1 has been extensively studied as an antigen for vaccine development against apicomplexan parasites, especially $P$. falciparum. Several antibodies, such as MAb 1F9 [30] and humAbAMA1 [32], and the invasion-inhibitory peptide R1 [31], raised against the conformational epitopes within the PAN motif of PfAMA-1 domain I, significantly inhibited the invasion of $P$. falciparum into human RBCs. Likewise, in the present study, the rabbit polyclonal antibody raised against the sBbAMA-1 epitope, which is located in the PAN motif of BbAMA-1 domain I and characterized by an a-helix secondary structure, significantly inhibited the growth (50-70\% inhibition) and invasion (60\% inhibition) of $B$. bovis merozoites. Our results are consistent with the previous studies [28] that showed the inhibitory effect of purified antibody against 
recombinant BbAMA-1 derived from the central region and ectodomains I and II. The purified IgG at the concentration of $1 \mathrm{mg} / \mathrm{ml}$ significantly inhibited the growth and invasion of $B$. bovis merozoites by approximately $40-50 \%$ and $60-70 \%$, respectively. Our results are also in agreement with the previous study in which a synthetic peptide derived from the N-terminal region of BbAMA-1 domain I inhibited the $B$. bovis growth (65\% inhibition) significantly [11]. Taken together, these results indicate that the epitope we predicted in the PAN domain I of BbAMA-1 is neutralization-sensitive. The antibodies against sBbAMA-1 may either block some specific functions of the AMA-1, involve in inhibition of proteolytic processing [11, 43] or block the interaction of AMA1 with its receptor [40]. The multiple alignment of BbAMA-1 sequences from various $B$. bovis isolates identified 6 polymorphic sites in the predicted epitope. Therefore, further studies are essential to investigate whether the observed polymorphism results in a strain-specific immunity.

\section{Conclusions}

The present study demonstrated that the rabbit polyclonal antibody against a synthetic peptide derived from the PAN motif of BbAMA-1 domain I is capable of inhibiting the in vitro growth and invasion of erythrocytes by $B$. bovis merozoites. Our data suggest that the predicted epitope or PAN motif of BbAMA1 domain I may serve as a target antigen for the development of vaccines against bovine babesiosis caused by $B$. bovis.

\section{Methods}

\section{In vitro cultivation of $B$. bovis}

The Texas (T2Bo) strain of B. bovis was maintained with purified bovine RBCs in serum-free GIT culture medium (WAKO Pure Chemical Industrial, Ltd., Osaka, Japan) supplemented with antibiotic-antimycotic solution (Sigma-Aldrich, Tokyo, Japan). Parasites were continuously cultured in an atmosphere of $5 \% \mathrm{O}_{2}$ and $5 \% \mathrm{CO}_{2}$ at $37^{\circ} \mathrm{C}$. The culture medium was changed daily [4].

\section{Prediction, analysis and synthesis of a B-cell epitope}

A BbAMA-1 amino acid sequence retrieved from UniProtKB database (entry no. A7ASF6) was subjected to bioinformatics analysis to predict both linear and conformational B-cell epitope in the PAN motif of domain I as described by Rittipornlertrak et al. (2017) [26]. The genetic diversity of the predicted epitope was visualized by multiply aligning the corresponding amino acid sequences from various $B$. bovis isolates, using the MUSCLE algorithm (http://www.ebi.ac.uk/Tools/msa/muscle/) [44]. In addition, a multiple alignment of complete AMA-1 sequences from Babesia spp. and other apicomplexan parasites was examined by I-TASSER server (https://zhanglab.ccmb.med.umich.edu/I-TASSER/) [45] for the comparative analysis of predicted secondary structures. Moreover, a homology model of the complete BbAMA-1 was constructed with Phyre2 (http://www.sbg.bio.ic.ac.uk/phyre2/html/page.cgi?id=index) [46] 
and I-TASSER server based on the 3D structure of Babesiadivergens (B. divergens) AMA-1 (BdAMA-1) [47]. The selected epitope was synthesized as a peptide (sBbAMA-1).

\section{Production of rabbit polyclonal anti-sBbAMA-1 antibody}

Two New Zealand White female rabbits (Mlac:NZW, National Laboratory Animal Center, Mahidol University, Nakhon Pathom, Thailand) with 3-6 months old were kept individually in cages without bedding at a temperature of $20^{\circ} \mathrm{C}$ to $24^{\circ} \mathrm{C}$, humidity of $55 \pm 10 \%$ and a $12 / 12$-hour light/dark cycle at laboratory building, Faculty of Veterinary Medicine, Chiang Mai University, Thailand. Rabbits were maintained with ad libitum access to food and water. To obtain rabbit polyclonal anti-sBbAMA-1 antibody, a rabbit was intramuscularly immunized with $100 \mu \mathrm{g} / \mathrm{ml} \mathrm{sBbAMA-1}$ formulated with Montanide ISA 206 VG (Seppic, Paris, France; 1:1 v/v) 4 times at 2-week interval after housing for 1 week. To obtain a negative control serum, the other rabbit was intramuscularly immunized with $0.5 \mathrm{ml}$ of Montanide adjuvant only. The adverse events were monitored throughout the experiment. Every week, serum samples were collected from the immunized rabbits to determine antibody titer using an indirect enzymelinked immunosorbent assay (ELISA). Briefly, each well in 96-well immuno plates (Nunc-Immuno Plate MaxiSorp, Intermed, Roskildes, Denmark) was coated with $10 \mathrm{ng}$ of sBbAMA-1 in a coating buffer ( $0.05 \mathrm{M}$ carbonate bicarbonate buffer, $\mathrm{pH}$ 9.6), and then incubated overnight at $4^{\circ} \mathrm{C}$. The plates were washed thrice with wash buffer (Phosphate-buffered saline (PBS) containing 0.05\% Tween-20) and then blocked with a blocking buffer (5\% skim milk in PBS). Rabbit serum samples at dilution of 1:100 in blocking buffer was added and then incubated at $37^{\circ} \mathrm{C}$ for $1 \mathrm{~h}$. After washing thrice with washing buffer, horseradish peroxidase (HRP)-conjugated goat anti-rabbit immunoglobulin (Ig) G (KPL, Gaithersburg, MD, USA) at dilution of $1: 2,000$ in blocking buffer was added and then incubated at $37^{\circ} \mathrm{C}$ for $1 \mathrm{~h}$. After washing thrice with washing buffer, 3,3',5,5'-tetramethybenzidine (TMB) substrate (SeraCare Life Sciences, Gaithersburg, MD, USA) was added, and incubated at room temperature in dark. The reaction was stopped with $2 \mathrm{M} \mathrm{H}_{2} \mathrm{SO}_{4}$ after 15 min of incubation. The absorbance at $450 \mathrm{~nm}$ was measured using an Accu Reader Microplate reader M965 (Metertech, Taipei, Taiwan R.O.C.). Due to the final blood collection of rabbits, the generalized anesthesia was done by intravenous injection of pentobarbitone sodium (Nembutal ${ }^{\circledR}, 20 \mathrm{mg} / \mathrm{kg}$ ). The blood collection was done by using a 1 -in. long, 18-gauge needle for penetrating to jugular vein. Blood was taken until the volume reached $100 \mathrm{ml}$, then the rabbits were euthanized by intravenous injection of overdosage pentobarbitone sodium (Nembutal ${ }^{\circledR}, 90 \mathrm{mg} / \mathrm{kg}$ ).

\section{Recognition of native BbAMA-1 by Western blot analysis}

The $B$. bovis lysate was prepared as described by Yokoyama et al. (2002) [48] with minor modifications. Briefly, the $B$. bovis infected RBCs from the in vitro cultures were treated with $0.83 \% \mathrm{NH}_{4} \mathrm{Cl}$ solution for 10 min at $37^{\circ} \mathrm{C}$, and then washed thrice with cold PBS. The pellets containing the parasites were suspended in $1 \mathrm{ml}$ of a lysis buffer (50 mM Tris- $\mathrm{HCl}$ (pH 7.6), 0.1\% Triton X-100, $150 \mathrm{mM} \mathrm{NaCl}, 20 \%$ glycerol, $1 \mathrm{mM}$ EDTA, $1 \mathrm{mM}$ phenylmethylsulfonyl fluoride (PMSF), $1 \mathrm{mM}$ dithiothreitol (DTT), $10 \mathrm{mg} / \mathrm{ml}$ pepstatin $A$ and $10 \mathrm{mg} / \mathrm{ml}$ leupeptin), incubated on ice for $20 \mathrm{~min}$, and then centrifuged at $18,000 \times \mathrm{g}$ for $30 \mathrm{~min}$ at $4^{\circ} \mathrm{C}$. The supernatant of clarified lysate was dialyzed overnight using a dialysis buffer $(50 \mathrm{mM}$ Tris- $\mathrm{HCl}(\mathrm{pH}$ 
7.6), $150 \mathrm{mM} \mathrm{NaCl}, 20 \%$ glycerol, $1 \mathrm{mM}$ EDTA, $1 \mathrm{mM}$ PMSF, and $1 \mathrm{mM}$ DTT). The dialysate was centrifuged at $18,000 \times \mathrm{g}$ for $30 \mathrm{~min}$ at $4^{\circ} \mathrm{C}$. Subsequently, the supernatant was analyzed to identify native BbAMA-1 by Western blot analysis. The lysate were separated by $12 \%$ sodium dodecyl sulfate polyacrylamide gel electrophoresis (SDS-PAGE) gel. The proteins from SDS-PAGE gel were electrically transferred onto a nitrocellulose membrane (Merck Millipore ${ }^{\mathrm{TM}}$, Merck KGaA ${ }^{\odot}$, Darmstadt, DEU). The blotting time was $60 \mathrm{~min}$ at a constant voltage of $20 \mathrm{~V}$. The membrane was then incubated with a blocking buffer (5\% skim milk in PBS) for $1 \mathrm{~h}$ at room temperature with gentle shaking. After being washed thrice with wash buffer (PBS containing $0.05 \%$ Tween ${ }^{\circledR} 20$ ), the membrane was incubated with rabbit polyclonal anti-sBbAMA-1 serum (1:50 dilution) at $4^{\circ} \mathrm{C}$ overnight. The membrane was probed with HRP-conjugated goat anti-rabbit IgG antibody (1:4,000 dilution; KPL, Gaithersburg, MD, USA). The membrane was incubated with gentle shaking at room temperature for $1 \mathrm{~h}$ and then washed three times with wash buffer. Finally, the reactions were visualized using a solution containing 3,3'-diaminobenzidine (DAB; Invitrogen, Carlsbad, $\mathrm{CA}, \mathrm{USA})$ and hydrogen peroxide $\left(\mathrm{H}_{2} \mathrm{O}_{2}\right.$; Merck, Germany).

\section{The immunofluorescence antibody test (IFAT)}

The immunofluorescence antibody test was carried out as described in a previous study [49] with minor modifications. The B. bovis-infected RBCs were coated on indirect IFAT slides (Matsunami Glass Ind., Ltd, Osaka, Japan), air-dried, and then fixed in cold absolute acetone at $-20^{\circ} \mathrm{C}$ for $5 \mathrm{~min}$. Ten microliters of the rabbit anti-sBbAMA-1 serum diluted at 1:40 in a blocking buffer ( $5 \%$ fetal bovine serum (FBS) in PBS) was added as the first antibody on the fixed smears, and then incubated for $1 \mathrm{~h}$ at $37^{\circ} \mathrm{C}$ in a moist chamber. After washing once with PBST, Alexa-FluorÒ 488 conjugated goat anti-rabbit IgG (Molecular Probes, Invitrogen, Carlsbad, CA, USA) was subsequently applied (1:200 dilution in the blocking buffer) as a secondary antibody, and then incubated for $30 \mathrm{~min}$ at $37^{\circ} \mathrm{C}$. The slides were washed thrice with PBST, mounted in 50\% glycerol-PBS, and then examined using a fluorescent microscope (E400 Eclipse, Nikon, Kawasaki, Japan).

\section{Preparation of free merozoites by cold treatment}

The method described by Ishizaki et al. (2016) [50] was used to isolate free merozoite liberated from the infected RBCs. A $5 \mathrm{ml}$ of an in vitro culture of B. bovis with $30 \%$ parasitemia was incubated on ice for $2 \mathrm{~h}$, and then the culture was resuspended in $5 \mathrm{ml}$ of GIT medium. The suspension was slowly overlaid onto 2 $\mathrm{ml}$ of $30 \%(1.043 \mathrm{~g} / \mathrm{ml}$ density) Percoll/ PBS solution (GE Healthcare, Buckinghamshire, UK) at the bottom of a $15 \mathrm{ml}$ centrifuge tube (Corning, Corning, NY, USA). The tube was centrifuged at $280 \mathrm{xg}$ for 5 min, and then at $330 \mathrm{xg}$ for $20 \mathrm{~min}$ at $4^{\circ} \mathrm{C}$. The medium and free merozoite layers were transferred carefully to a new tube, and then centrifuged at $1500 \mathrm{xg}$ for $5 \mathrm{~min}$ at $4^{\circ} \mathrm{C}$. The pellet containing free merozoites was washed twice with $20 \mathrm{ml}$ of GIT medium, and then suspended in $1 \mathrm{ml}$ of GIT medium. The concentration of purified merozoites was calculated with a disposable hemo-cytometer (AR Brown, Tokyo, Japan). The viability of the merozoites was determined after staining with 6-carboxyfluorescein diacetate (6-CFDA; Invitrogen Corp., Carlsbad, CA, USA) and propidium iodide (PI; Dojindo, Kumamoto, Japan). 


\section{In vitro growth-inhibition assay}

The growth-inhibition assay was performed in 96-well culture plates (Nunc, Roskilde, Denmark) as described by Salama et al. (2013) [28]. One hundred and eighty microliters of GIT medium, GIT medium containing anti-sBbAMA-1 serum at 1:5, 1:10, and 1:20 or rabbit anti-Montanide adjuvant serum (negative control) at 1:5 dilution was added into each well in triplicates. Then, $20 \mu \mathrm{l}$ of bovine RBCs with $1 \%$ parasitemia was added into each well. The plate was incubated as described in section 2.1 for 4 days, and the culture medium was replaced everyday with fresh medium containing the indicated dilution of antiserum. Giemsa-stained thin blood smears were prepared every $24 \mathrm{~h}$ and parasitemia was monitored by counting the infected RBCs among approximately 1,000 total RBCs, using a light microscope. The percentage of growth inhibition was calculated as the rate of parasitemia reduction in antibody-treated cultures compared to GIT-control. The assay was repeated twice.

\section{In vitro invasion-inhibition assay}

The RBC invasion-inhibition assay was performed in triplicate in a 96-well plates (Nunc, Roskilde, Denmark) according to Ishizaki et al. (2016) [50] with some modification. Briefly, purified free merozoites (obtained from $B$. bovis in vitro culture) together with uninfected bovine RBCs with a multiplicity of infection (MOI) of 4.2 were added to GIT medium containing rabbit anti-sBbAMA-1 serum at 1:5 and 1:10 dilutions. Rabbit anti-Montanide adjuvant serum at dilution of 1:5 and GIT medium were used as control. The culture plate was incubated, and Giemsa-stained thin blood smears were prepared at 1, 2 and $4 \mathrm{~h}$ post-incubation. The percentage of parasitemia was evaluated under a light microscope, based on approximately 3,000 observed RBCs. The percentage of invasion-inhibition was calculated based on the parasitemia reduction in antibody-treated cultures compared to GIT-control. The experiments were carried out in two separate trials.

\section{Statistical analyses}

The growth- and invasion-inhibition rates were analyzed by independent-samples Student's $t$ test. $P$ values $<0.05$ were considered statistically significant.

\section{Abbreviations}

AMA-1: Apical membrane antigen 1; $\mathrm{NH}_{4} \mathrm{Cl}$; Ammonium chloride; $B$. divergens.Babesia divergens; BdAMA1:B. divergens AMA-1; B. bovis:Babesia bovis, BbAMA-1:B. bovis AMA-1; 6-CFDA:6-carboxyfluorescein diacetate; DAB:3,3'-diaminobenzidine; DTT:Dithiothreitol; EDTA:Ethylene diamine tetraacetic acid; ELISA:Enzyme-linked immunosorbent assay; FBS:Fetal bovine serum; $\mathrm{HCl}$ :Hydrochloric acid; HRP:Horseradish peroxidase; $\mathrm{H}_{2} \mathrm{O}_{2}$ :Hydrogen peroxide; IFAT:Immunofluorescence antibody test; Ig:Immunoglobulin; MIC:Microneme protein; MOI:Multiplicity of infection; PMSF:Phenylmethylsulfonyl fluoride; PAN:Plasminogen, apple and nematode; PBS:Phosphate-buffered saline; PBST:Phosphatebuffered saline containing 0.05\% Tween ${ }^{\circledR} 20 ;$; f falciparum:Plasmodium falciparum; PfAMA-1:P. falciparum AMA-1; PfRON2:P. falciparum RON; PI:Propidium iodide; RBCs:Red blood cells; RON:Rhoptry 
neck proteins; NaCl:Sodium chloride; SDS-PAGE:Sodium dodecyl sulfate polyacrylamide gel electrophoresis; sBbAMA-1:synthetic BbAMA-1 peptide; $\mathrm{H}_{2} \mathrm{SO}_{4}$ : Sulfuric acid; TMB:Tetramethylbenzidine;

\section{Declarations}

\section{Ethics approval and consent to participate}

All animal protocols in this study were approved and supervised by the Animal Care and Use Committee (FVM-ACUC), Faculty of Veterinary Medicine, Chiang Mai University (Approval No. S28/2560).

\section{Consent for publication}

Not applicable.

\section{Availability of data and materials}

The datasets used and/or analysed during the current study are available from the corresponding author on reasonable request.

\section{Competing interests}

The authors declare that they have no competing interest.

\section{Funding}

This work was supported by the Royal Golden Jubilee Ph.D. Programme scholarship (grant no. PHD/0224/2560). Additionally, this work was also funded by the Center of Excellence for Veterinary Bioscience, Chiang Mai University (grant No. 018/2562). Funding body had no role in the design of the study, collection, analysis, and interpretation of data and in writing the manuscript.

\section{Authors' contributions}

Conceived and designed the experiment: AR, VP, ST, BT, TS, NY and NS.

Performed the experiment: AR, BN, AM, BT

Analyzed the data: AR, BT, TS

Wrote the paper: AR

Reviewed and edited the paper: VP, ST, YTC, NY and NS

All authors have read and approved of the final version of the manuscript for submission and publication. 
We would like to express our deep appreciation to all staffs of the Babesia group, National Research Center for Protozoan Diseases, Obihiro University of Agriculture and Veterinary Medicine, Hokkaido, Japan for materials, facilities and technical assistance. Mr. Amarin Rittipornlertrak is a PhD student under the Royal Golden Jubilee Ph.D. Programme scholarship (grant no. PHD/0224/2560).

\section{References}

1. Spickler AR. Bovine Babesiosis. 2018. http://www.cfsph.iastate.edu/Diseaselnfo/fact sheets.php:. Accessed 13 July 2020.

2. Bock R, Jackson L, De Vos A, Jorgensen W. Babesiosis of cattle. Parasitology. 2004;129:S247.

3. Gohil S, Herrmann S, Günther S, Cooke BM. Bovine babesiosis in the $21^{\text {st }}$ century: advances in biology and functional genomics. Int J Parasitol. 2013;43:125-32.

4. Tuvshintulga B, Sivakumar T, Yokoyama N, Igarashi I. Development of unstable resistance to diminazene aceturate in Babesia bovis. Int J Parasitol Drugs Drug Resist. 2019;9:87-92.

5. Moreau E, Bonsergent C, Al Dybiat I, Gonzalez LM, Lobo CA, Montero E, et al. Babesia divergens apical membrane antigen-1 (BdAMA-1): A poorly polymorphic protein that induces a weak and late immune response. Exp Parasitol. 2015;155:40-5.

6. De Waal DT, Combrink MP. Live vaccines against bovine babesiosis. Vet Parasitol. 2006;138:88-96.

7. De Vos AJ, Bock RE. Vaccination against bovine babesiosis. Ann N Y Acad Sci. 2000;916:540-5.

8. Shkap V, de Vos AJ, Zweygarth E, Jongejan F. Attenuated vaccines for tropical theileriosis, babesiosis and heartwater: the continuing necessity. Trends Parasitol. 2007;23:420-6.

9. Suarez CE, Alzan HF, Silva MG, Rathinasamy V, Poole WA, Cooke BM. Unravelling the cellular and molecular pathogenesis of bovine babesiosis: is the sky the limit?. Int J Parasitol. 2019;49:183-97.

10. Remarque EJ, Faber BW, Kocken $\mathrm{CH}$, Thomas AW. Apical membrane antigen 1: a malaria vaccine candidate in review. Trends Parasitol. 2008;24:74-84.

11. Gaffar FR, Yatsuda AP, Franssen FFJ, de Vries E. Erythrocyte invasion by Babesia bovis merozoites is inhibited by polyclonal antisera directed against peptides derived from a homologue of Plasmodium falciparum apical membrane antigen 1. Infect Immun. 2004;72:2947-55.

12. Triglia T, Healer J, Caruana SR, Hodder AN, Anders RF, Crabb BS, et al. Apical membrane antigen 1 plays a central role in erythrocyte invasion by Plasmodium species. Mol Microbiol. 2000;38:706-18.

13. Yap A, Azevedo MF, Gilson PR, Weiss GE, O'Neill MT, Wilson DW, et al. Conditional expression of apical membrane antigen 1 in Plasmodium falciparum shows it is required for erythrocyte invasion by merozoites. Cell Microbiol. 2014;16:642-56.

14. Srinivasan P, Beatty WL, Diouf A, Herrera R, Ambroggio X, Moch JK, et al. Binding of Plasmodium merozoite proteins RON2 and AMA1 triggers commitment to invasion. Proc Natl Acad Sci. 2011;108:13275-80.

15. Hodder AN, Crewther PE, Anders RF. Specificity of the protective antibody response to apical membrane antigen 1. Infect Immun. 2001;69:3286-94. 
16. Dutta S, Lalitha PV, Ware LA, Barbosa A, Moch JK, Vassell MA, et al. Purification, characterization, and immunogenicity of the refolded ectodomain of the Plasmodium falciparum apical membrane antigen 1 expressed in Escherichia coli. Infect Immun. 2002;70:3101-10.

17. Malkin EM, Diemert DJ, McArthur JH, Perreault JR, Miles AP, Giersing BK, et al. Phase 1 clinical trial of apical membrane antigen 1: an asexual blood-stage vaccine for Plasmodium falciparum malaria. Infect Immun. 2005;73:3677-85.

18. Srinivasan P, Baldeviano GC, Miura K, Diouf A, Ventocilla JA, Leiva KP, et al. A malaria vaccine protects Aotus monkeys against virulent Plasmodium falciparum infection. npj Vaccines. 2017;2:110.

19. Stowers AW, Kennedy MC, Keegan BP, Saul A, Long CA, Miller LH. Vaccination of monkeys with recombinant Plasmodium falciparum apical membrane antigen 1 confers protection against bloodstage malaria. Infect Immun. 2002;70:6961-7.

20. Dicko A1, Diemert DJ, Sagara I, Sogoba M, Niambele MB, Assadou MH. Impact of a Plasmodium falciparum AMA1 vaccine on antibody responses in adult Malians. PLoS ONE. 2007;2:e1045.

21. Sirima SB, Durier C, Kara L, Houard S, Gansane A, Loulergue P. Safety and immunogenicity of a recombinant Plasmodium falciparum AMA1-DiCo malaria vaccine adjuvanted with GLA-SE or Alhydrogel $\circledast$ in European and African adults: a phase 1a/1b, randomized, double-blind multi-centre trial. Vaccine. 2017;35:6218-27.

22. Thera MA, Doumbo OK, Coulibaly D, Laurens MB, Kone AK, Guindo AB. Safety and immunogenicity of an AMA1 malaria vaccine in Malian children: results of a phase 1 randomized controlled trial. PLoS ONE. 2010;5:e9041.

23. Drew DR, Hodder AN, Wilson DW, Foley M, Mueller I, Siba PM. Defining the antigenic diversity of Plasmodium falciparum apical membrane antigen 1 and the requirements for a multi-allele vaccine against malaria. PLoS ONE. 2012;7:e51023.

24. Escalante AA, Grebert HM, Chaiyaroj SC, Magris M, Biswas S, Nahlen BL. Polymorphism in the gene encoding the apical membrane antigen-1 (AMA-1) of Plasmodium falciparum. X. Asembo Bay Cohort Project. Mol Biochem Parasitol. 2001;113:279-87.

25. Ramos CA, Araújo FR, Alves LC, de Souza IIF, Guedes Jr DS, Soares CO. Genetic conservation of potentially immunogenic proteins among Brazilian isolates of Babesia bovis. Vet Parasitol. 2012;187:548-52.

26. Rittipornlertrak A, Nambooppha B, Simking P, Punyapornwithaya V, Tiwananthagorn S, Jittapalapong $\mathrm{S}$, et al. Low levels of genetic diversity associated with evidence of negative selection on the Babesia bovis apical membrane antigen 1 from parasite populations in Thailand. Infect Genet Evol. 2017;54:447-54.

27. Barreda D, Hidalgo-Ruiz M, Hernandez-Ortiz R, Ramos JA, Galindo-Velasco E, Mosqueda J. Identification of conserved peptides containing B-cell epitopes of Babesia bovis AMA-1 and their potential as diagnostics candidates. Transbound Emerg Dis. 2019;00:1-9. 
28. Salama AA, Terkawi MA, Kawai S, Aboulaila M, Nayel M, Mousa A, et al. Specific antibody to a conserved region of Babesia apical membrane antigen-1 inhibited the invasion of $B$. bovis into the erythrocyte. Exp Parasitol. 2013;135:623-8.

29. Coley AM, Campanale NV, Casey JL, Hodder AN, Crewther PE, Anders RF, et al. Rapid and precise epitope mapping of monoclonal antibodies against Plasmodium falciparum AMA1 by combined phage display of fragments and random peptides. Protein Eng. 2001;14:691-8.

30. Coley AM, Parisi K, Masciantonio R, Hoeck J, Casey JL, Murphy VJ, et al. The most polymorphic residue on Plasmodium falciparum apical membrane antigen 1 determines binding of an invasion inhibitory antibody. Infect Immun. 2006;74:2628-36.

31. Harris KS, Casey JL, Coley AM, Masciantonio R, Sabo JK, Keizer DW, et al. Binding hot spot for invasion inhibitory molecules on Plasmodium falciparum apical membrane antigen 1. Infect Immun. 2005;73:6981-9.

32. Maskus DJ, Królik M, Bethke S, Spiegel H, Kapelski S, Seidel M, et al. Characterization of a novel inhibitory human monoclonal antibody directed against Plasmodium falciparum apical membrane antigen 1. Sci Rep. 2016;6:39462.

33. Pizarro JC, Vulliez-Le Normand B, Chesne-Seck ML, Collins CR, Withers-Martinez C, Hackett F, et al. Crystal structure of the malaria vaccine candidate apical membrane antigen 1 . Science. 2005;308:408-11.

34. Coley AM, Gupta A, Murphy VJ, Bai T, Kim H, Anders RF, et al. Structure of the malaria antigen AMA1 in complex with a growth-inhibitory antibody. PLoS Pathog. 2007;3:1308-19.

35. Richard D, MacRaild CA, Riglar DT, Chan JA, Foley M, Baum J, et al. Interaction between Plasmodium falciparum apical membrane antigen 1 and the rhoptry neck protein complex defines a key step in the erythrocyte invasion process of malaria parasites. J Biol Chem. 2010;285:14815-22.

36. Vulliez-Le Normand B, Tonkin ML, Lamarque MH, Langer S, Hoos S, Roques M, et al. Structural and functional insights into the malaria parasite moving junction complex. PLoS Pathog. 2012;8:e1002755.

37. Lim SS, Yang W, Krishnarjuna B, Kannan Sivaraman K, Chandrashekaran IR, Kass I, et al. Structure and dynamics of apical membrane antigen 1 from Plasmodium falciparum FVO. Biochemistry. 2014;53:7310-20.

38. Bai T, Becker M, Gupta A, Strike P, Murphy VJ, Anders RF, et al. Structure of AMA1 from Plasmodium falciparum reveals a clustering of polymorphisms that surround a conserved hydrophobic pocket. Proc Natl Acad Sci. 2005;102:12736-41.

39. Howell SA, Withers-Martinez C, Kocken $\mathrm{CH}$, Thomas AW, Blackman MJ. Proteolytic processing and primary structure of Plasmodium falciparum apical membrane antigen-1. J Biol Chem. 2001;276:31311-20.

40. Montero E, Rodriguez M, Oksov Y, Lobo CA. Babesia divergens apical membrane antigen 1 and its interaction with the human red blood cell. Infect Immun. 2009;77:4783-93. 
41. Moitra P, Zheng H, Anantharaman V, Banerjee R, Takeda K, Kozakai Y, et al. Expression, purification, and biological characterization of Babesia microti apical membrane antigen 1. Infect Immun. 2015;83:3890-3901.

42. AbouLaila M, Terkawi M, Menshawy S, Yokoyama N, Igarashi I. Identification, characterization, and protective effect of the apical membrane antigen-1 (AMA-1) Homolog from the Argentina Strain of Babesia bigemina. Ann Clin Investig. 2019;1:1001.

43. Dutta S, Haynes JD, Moch JK, Barbosa A, Lanar DE. Invasion-inhibitory antibodies inhibit proteolytic processing of apical membrane antigen 1 of Plasmodium falciparum merozoites. Proc Natl Acad Sci. 2003;100:12295-300.

44. Edgar RC. MUSCLE: a multiple sequence alignment method with reduced time and space complexity. BMC bioinformatics. 2004;5:113.

45. Yang J, Zhang Y. I-TASSER server: new development for protein structure and function predictions. Nucleic Acids Res. 2015;43:174-81.

46. Kelley LA, Mezulis S, Yates CM, Wass MN, Sternberg MJ. The Phyre2 web portal for protein modeling, prediction and analysis. Nat Protoc. 2015;10:845.

47. Tonkin ML, Crawford J, Lebrun ML, Boulanger MJ. Babesia divergens and Neospora caninum apical membrane antigen 1 structures reveal selectivity and plasticity in apicomplexan parasite host cell invasion. Protein Sci. 2013;22:114-27.

48. Yokoyama N, Suthisak B, Hirata H, Matsuo T, Inoue N, Sugimoto C, et al. Cellular localization of Babesia bovis merozoite rhoptry-associated protein 1 and its erythrocyte-binding activity. Infect Immun. 2002;70:5822-6.

49. Terkawi MA, Huyen NX, Shinuo C, Inpankaew T, Maklon K, Aboulaila M, et al. Molecular and serological prevalence of Babesia bovis and Babesia bigemina in water buffaloes in the northeast region of Thailand. Vet Parasitol. 2011;178:201-7.

50. Ishizaki T, Sivakumar T, Hayashida K, Tuvshintulga B, Igarashi I, Yokoyama N. RBC invasion and invasion-inhibition assays using free merozoites isolated after cold treatment of Babesia bovisin vitro culture. Exp Parasitol. 2016;166:10-15.

\section{Figures}


A)

T2BO-USA TECOMAN-MEXICO SOUTHEAST-BRAZIL THAILAND3-THAILAND GON-ISRAEL P32-SRI LANKA MH-ISRAEL
181 KTRGSSSVIAAKLSPVSAKDIRRWGYZGNDVANCSEYASNI IPASDKTTKYRYPFVFDSD 240 181 KTRGSSSVTAAKLSPVSAKDLRRWGYBGNDVANCSEYASNIIPASDKTTKYRYPFVEDSD 240 181 KTRGSSSVTAAKLSPVSAKDLRRWGYBGNDVANCSEYASNIIPASDKTTKYRYPFVFDSD 240 181 KTRGSSSVTAAKLSPVSAKDLRRWGYBGNDVANCSEYASNLIPASDKTTKYRYPFVFDSD 240 181 KTRGSSSASAAKLSPVSARDLRRWGYEGNDVANCSEYASNIIPASDRSTKYRYPFVEDSD 240 181 KTRGSSSASAAKLSPVSAKDLRRWGYZGNDVANCSEYASNIIPASDRSTKYRYPFVFDSD 240 181 KTRGSSSASAAKLSPVSAKDLRRWGYEGNDVANCSEYASNHIPASDRSTKYRYPFVEDSD 240

B)
Struct1
Struct2
Struct3
N. caninum
T. gondii
P. falciparum
P. vivax
B. bovis
B. divergens

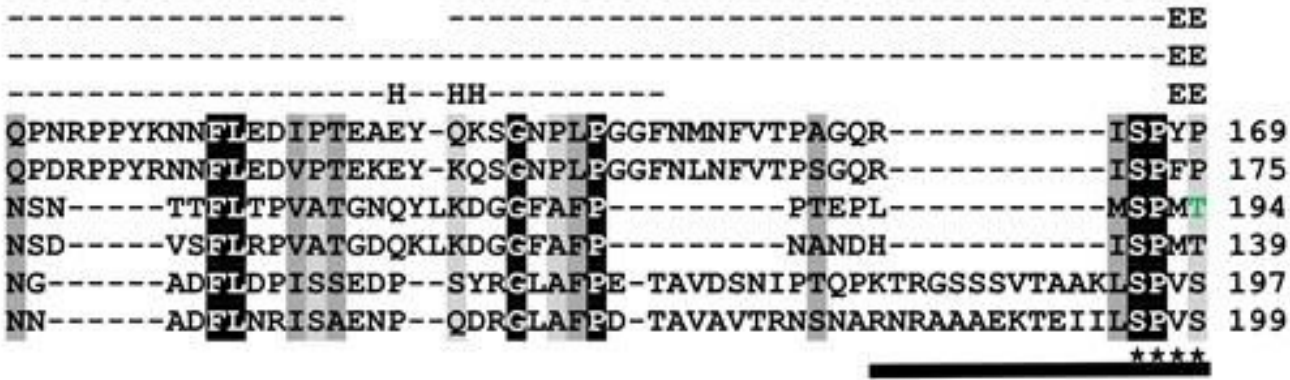

Struct1

Struct2

Struct3

N. caninum

T. gondii

P. falciparum

P. vivax

B. bovis

B. divergens

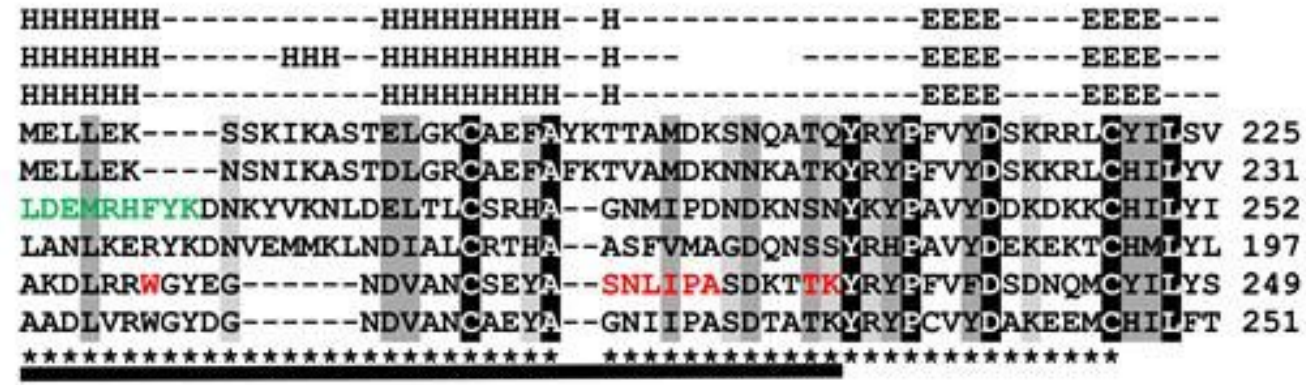

Struct1

Struct2

Struct3

N. caninum

T. gondii

P. falciparum

P. vivax

B. bovis

B. divergens

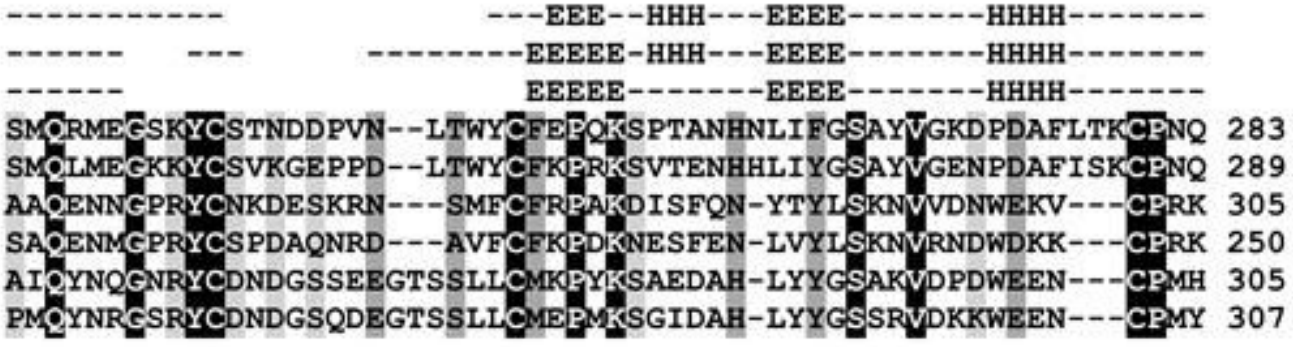

\section{Figure 1}

Sequence alignment of the BbAMA-1 with those sequences available in data base. A) An alignment showing polymorphisms in the epitope predicted in the PAN motif of BbAMA-1 domain I (181-230) using immunoinformatics tools. The following sequences from GenBank were used to generate the alignment; XP_001611043, ASR91672, ACM44020, BAN78782, ANW86202, ANW86201 and QDK56771. The epitope region is highlighted in blue. B) Alignment of the apicomplexan AMA-1 sequences correspond to PAN motif of PfAMA-1 domain I [38] from various apicomplexan parasite species. The GenBank-derived AMA1 sequences of Plasmodium vivax (Q9TY14), P. falciparum (Q7KQK5), Neospora caninum (B6KAM0), Toxoplasma gondii (A2A114), Babesia divergens (COIR59) and B. bovis (A7ASF6) were used in the alignment. The aligned secondary structure of AMA-1 from P. falciparum (Struct1, PDB: 4r1b), P. vivax 
(struct2, PDB: 1w8k) and B. divergens (Struct3, PDB: 4apm) are indicated as " $\mathrm{E}$ " for $\triangle$ strand and " $\mathrm{H}$ " for $\square$ helix. The epitope predicted in this study is indicated by a black bar. The epitope mapped by MAb 1F9 is indicated by asterisks (*). The MHC-restricted CD8+ T cell epitope in P. falciparum is highlighted with green letters. The residues associated with the invasion-inhibitory peptide R1 predicted by I-TASSER are highlighted with red letters.

A)
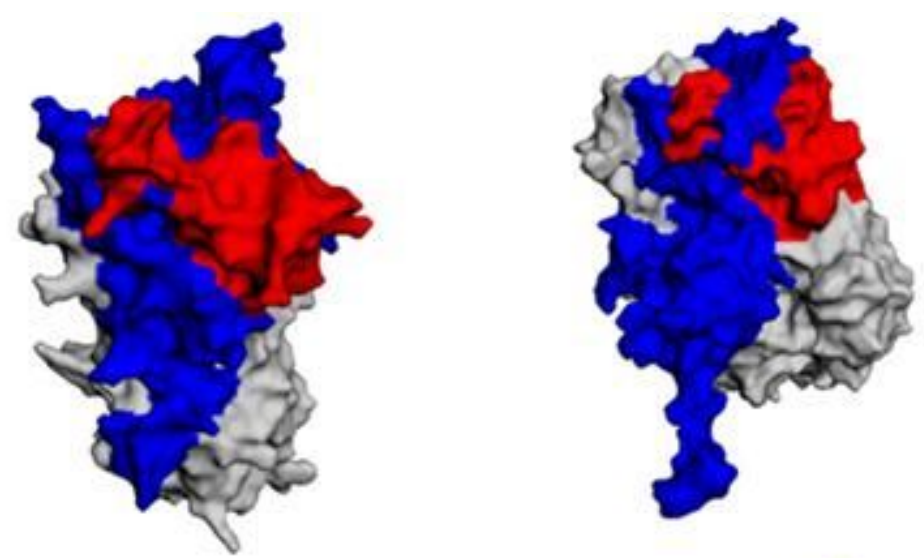

B)

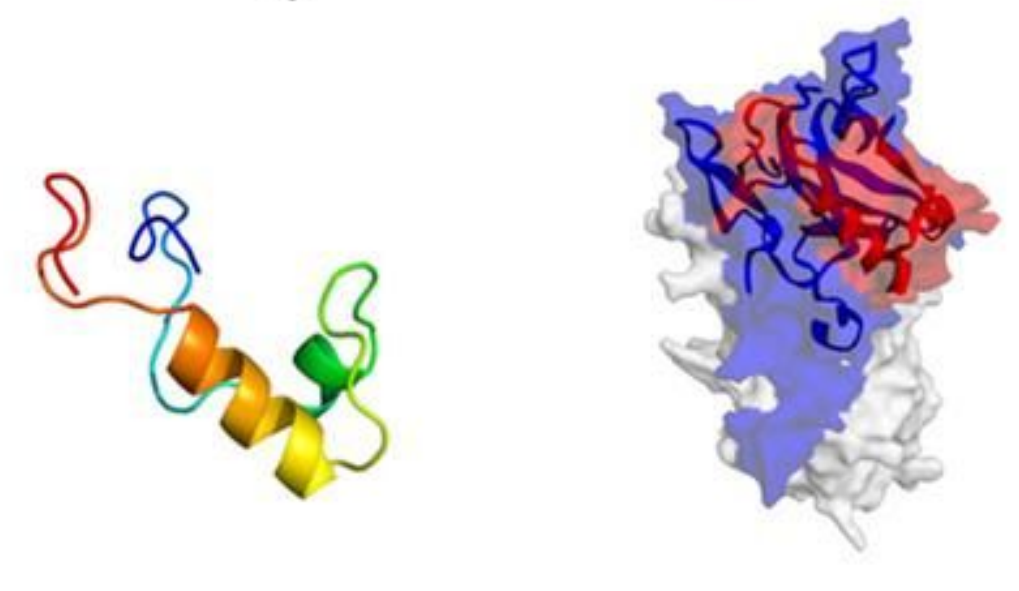

C)
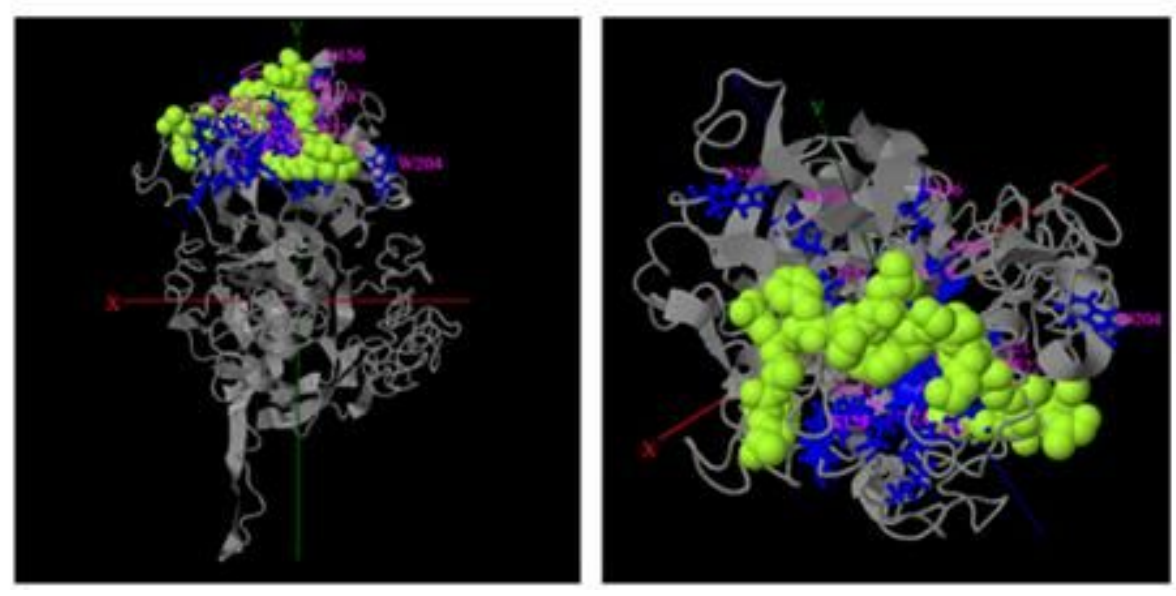

Figure 2

Homology modelling of BbAMA-1. A) A homology model of the complete BbAMA-1 was constructed based on BdAMA-1 using Phyre2 and I-TASSER (Left). The final model of BbAMA-1 was construct by ITASSER (Right). The structure showed the domain I of BbAMA-1 are represented by blue which contains the epitope areas predicted in this study indicated by red. The domain II-III are represented by grey. B) The 


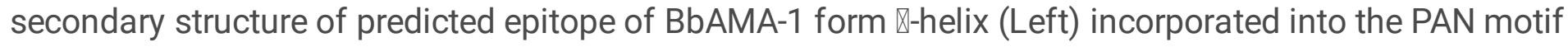
of BbAMA-1 domain I represented by red based on BdAMA-1 structure (Right). C) The invasion-inhibitory peptide R1 (PDB: 3srj, green atoms) associated predicted epitope residues (blue) analyzed by I-TASSER. The grey color structure is the BbAMA-1 constructed by I-TASSER.

A)

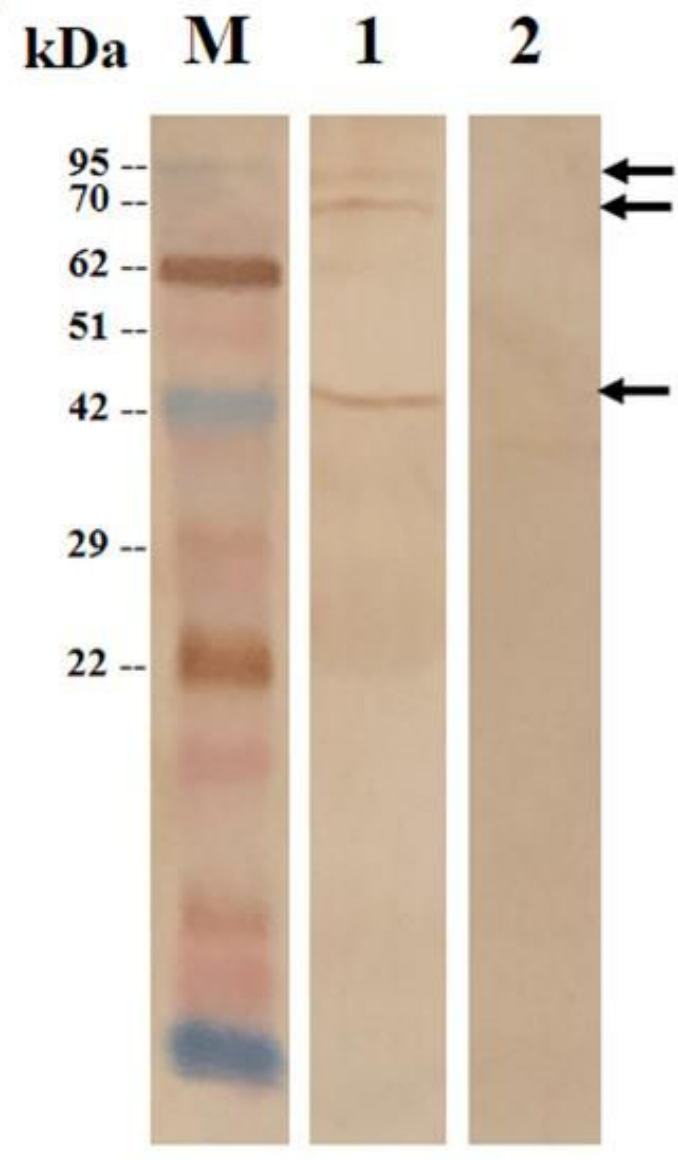

B)
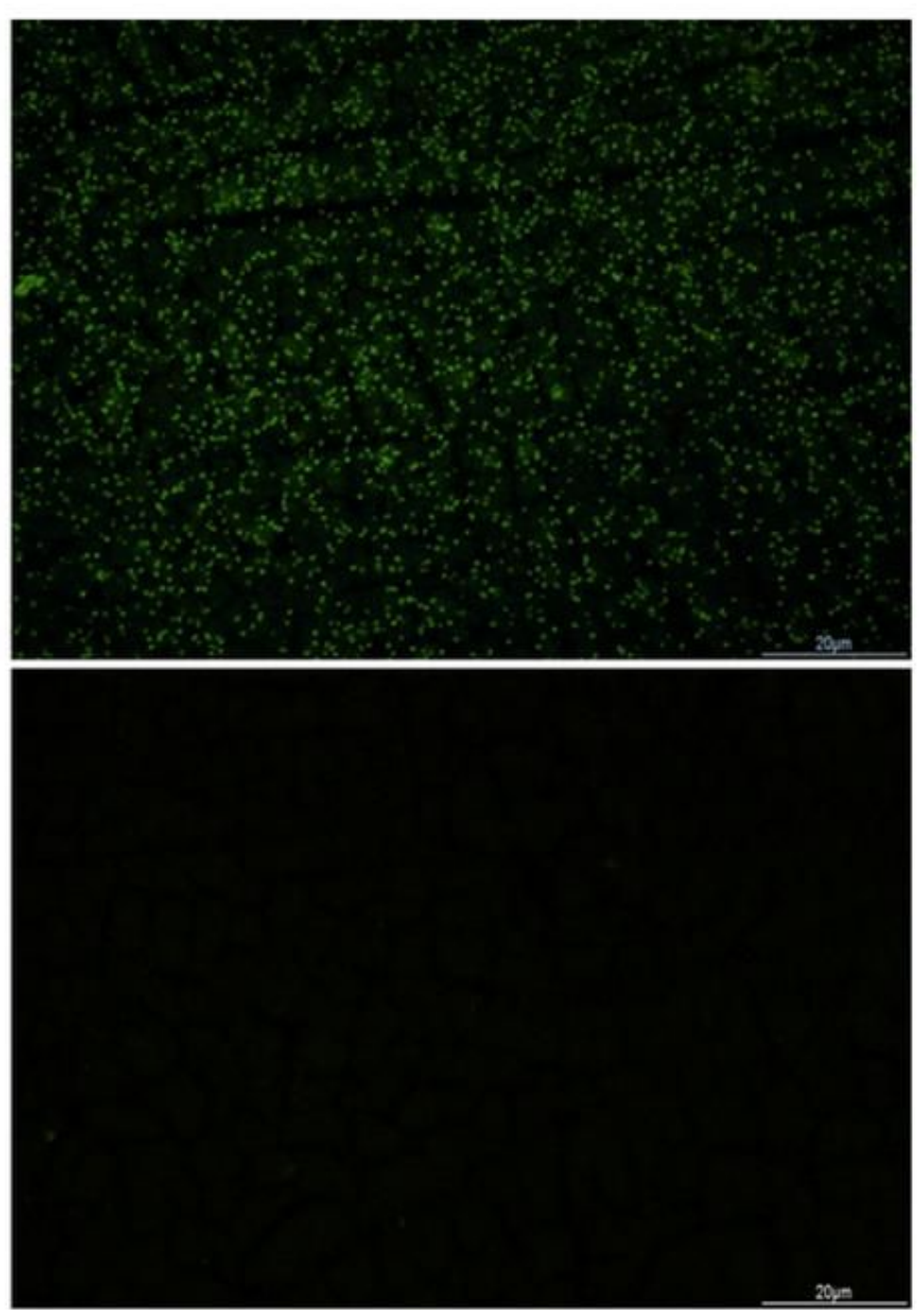

\section{Figure 3}

Recognition of native BbAMA-1 protein by rabbit polyclonal anti-sBbAMA-1 antibody. A) Western blot assay was conducted to assess the reactivity of anti-sBbAMA-1 antibody with the native BbAMA-1. Lane M; Marker, Lane 1; B. bovis-infected RBC lysate; lane 2; non-infected RBC lysate. B) The immunofluorescence antibody test was performed to confirm the binding of sBbAMA-1 to the surface of B. bovis merozoites. The rabbit polyclonal anti-sBbAMA-1 serum (upper panel) or rabbit negative serum (lower panel) was incubated with B. bovis-infected RBSs at 1:5 dilution. 


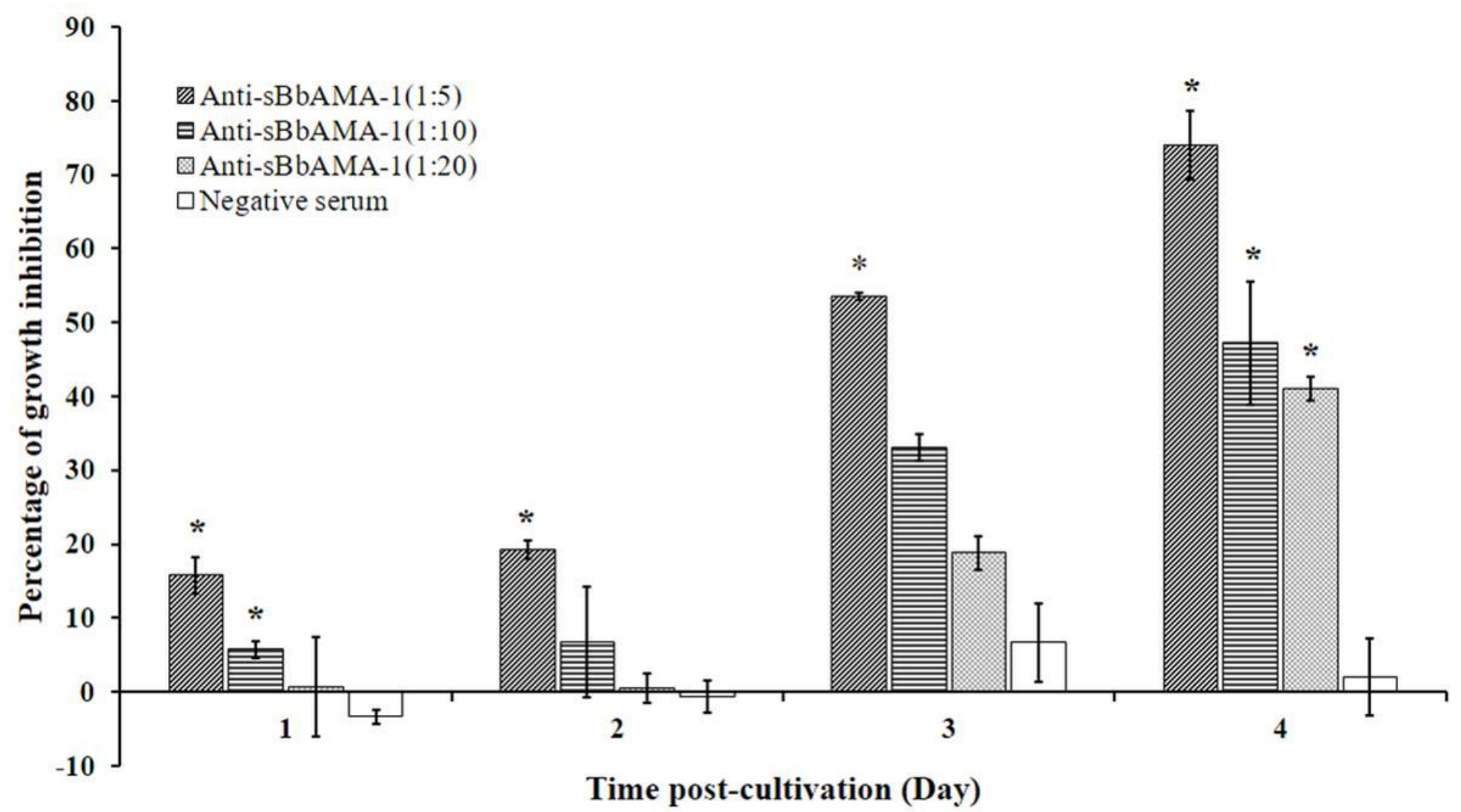

Figure 4

The growth-inhibitory effect of rabbit anti-sBbAMA-1 antibody on B. bovis merozoites. The in vitro growth inhibition assay was conducted with different dilution of anti-sBbAMA-1 serum and rabbit negative serum. The percentage of growth inhibition was calculated based on the parasitemia reduction in treated cultures compared to GIT-control. Asterisk indicates a significant difference $(p<0.05)$ in the percentage of growth inhibition in anti-sBbAMA-1 treated cultures compared to negative serum control.
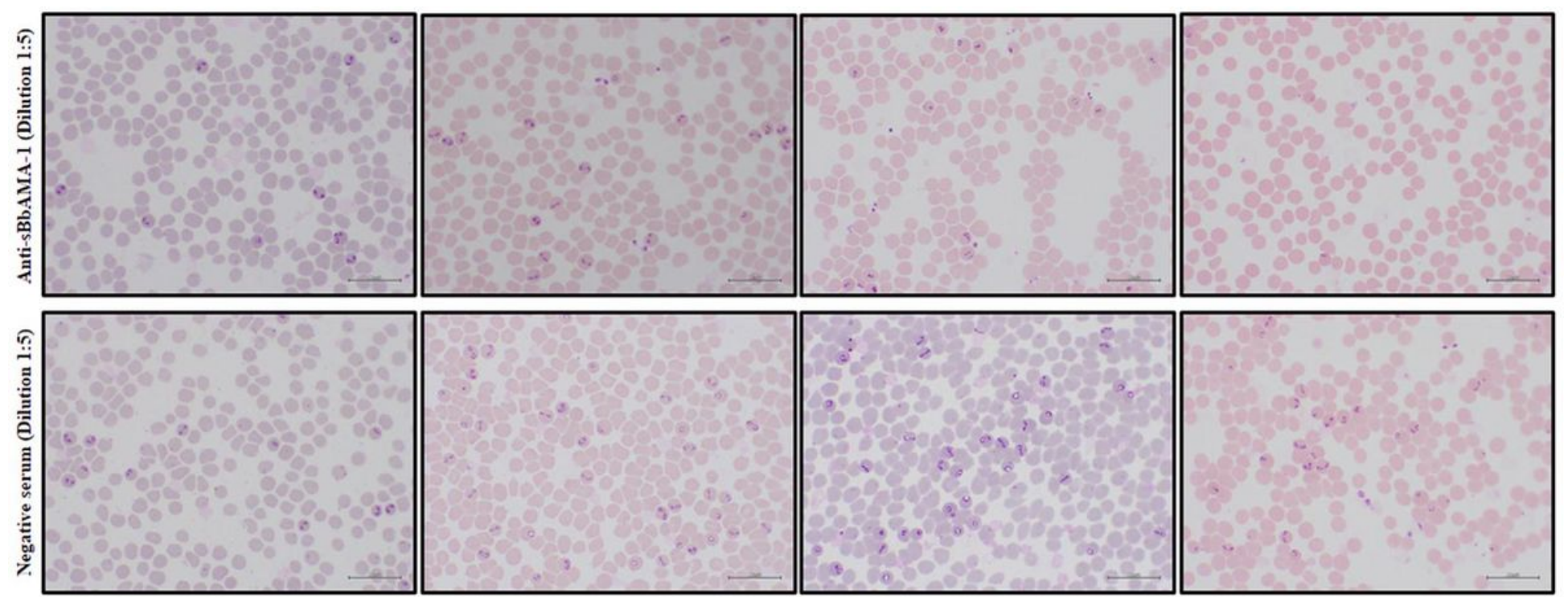

D1

D2

D3

D4

Figure 5 
Light microscopic examination of blood smears from in vitro cultures treated with rabbit polyclonal antisBbAMA-1 antibody. Blood smears prepared with the B. bovis-infected RBCs from cultures treated with anti-sBbAMA-1 serum (upper panel) or rabbit negative serum (lower panel) at 1:5 dilution were observed under a light microscope. D1, D2, D3 and D4 indicate days 1, 2, 3 and 4 post-cultivation. Note the presence of pyknotic extracellular merozoites in the treated cultures.

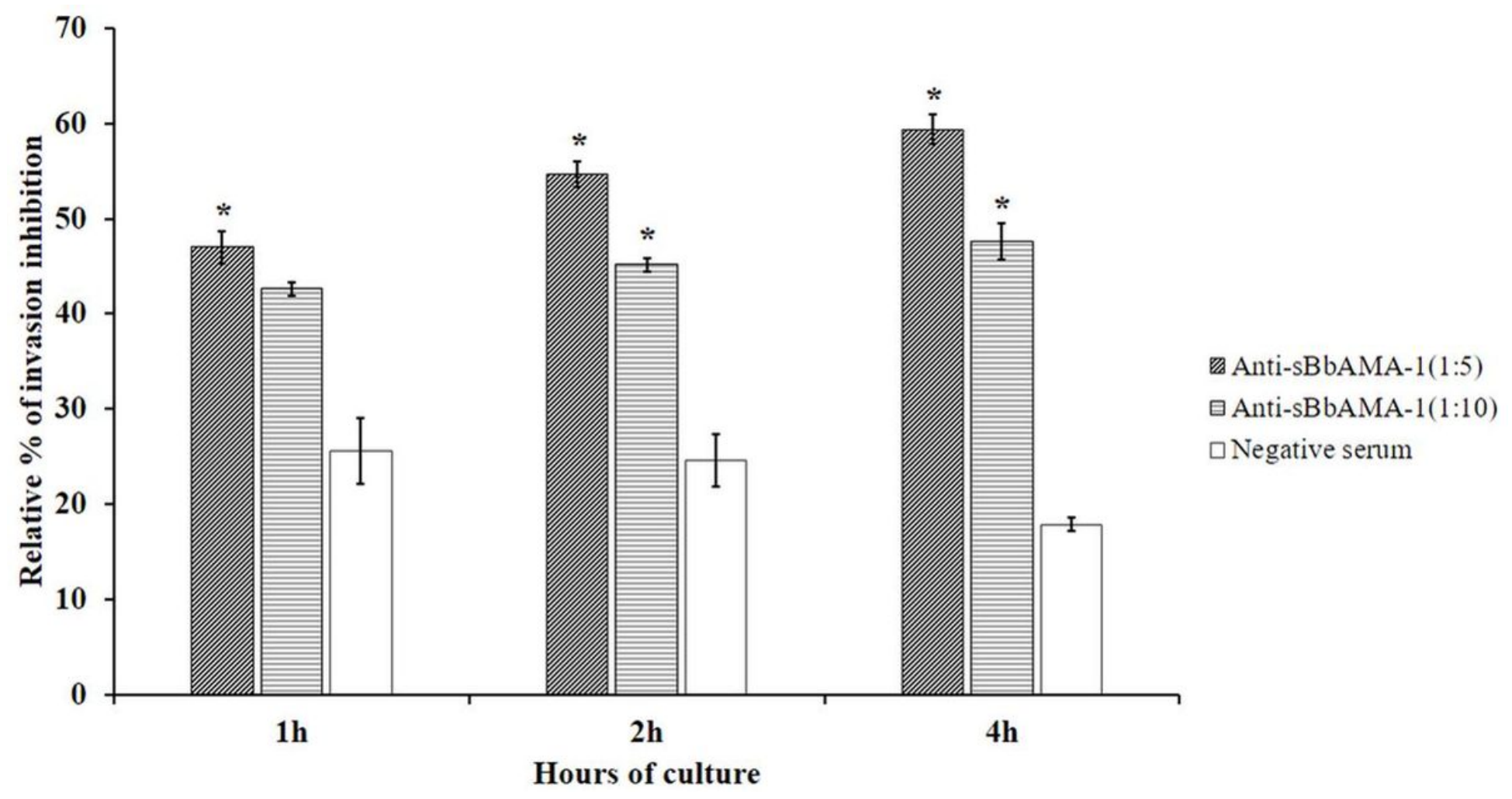

\section{Figure 6}

The evaluation of the inhibitory effect of rabbit anti-sBbAMA-1 antibody against $\mathrm{B}$. bovis merozoite invasion. Purified B. bovis merozoites were incubated together with fresh bovine RBCs in GIT medium containing rabbit anti-sBbAMA-1 serum at 1:5 and 1:10 dilution or rabbit negative serum at 1:5 dilutions. The percentage of invasion inhibition was calculated based on the parasitemia reduction in anti-sBbAMA1 antibody-treated cultures compered to GIT-control. Asterisk indicates a significant difference $(p<0.05)$ compared to negative serum control.

\section{Supplementary Files}

This is a list of supplementary files associated with this preprint. Click to download.

- RittipornlertraketalAdditionalfile1.pdf

- RittipornlertraketalAdditionalfile21.docx 